\title{
Monocular UHECR Spectra as Measured by HiRes
}

\author{
D. R. Bergman ${ }^{\text {a }}$ presented on behalf of the High Resolution Fly's Eye Collaboration \\ ${ }^{a}$ Rutgers, The State Univeristy of New Jersey \\ Department of Physics and Astronomy \\ Piscataway, New Jersey, USA 08854
}

We have measured the spectrum of UHE cosmic rays in monocular mode using separately both detectors the High Resolution Fly's Eye experiment. We describe the two detectors and the basic methods of analysis, and we present our measured spectra. We compare these spectra with that produced by an astrophysical source model with galactic and uniformly distributed extra-galactic sources. We also compare our spectra to the spectra produced by the AGASA experiment.

\section{Detectors}

The HiRes experiment consists of two airfluorescence detectors separated by $12.6 \mathrm{~km}$ and located at the U.S. Army Dugway Proving Ground in Utah. One detector, HiRes-I, consists of 22 mirrors covering $3-17^{\circ}$ in elevation and nearly $360^{\circ}$ in azimuth 1 . It uses a sample-andhold data acquisition system to save the photomultiplier tubes' pulse height and time information. HiRes-I has been running since June of 1997. The second detector, HiRes-II, located 12.6 $\mathrm{km}$ away, consists of 42 mirrors, covers $3-31^{\circ}$ in elevation, and uses a flash ADC (FADC) system to save pulse height and time information from its phototubes [2]. The sampling period of the FADC electronics is $100 \mathrm{~ns}$. HiRes-II has been operating since October of 1999. The mirror units at each site are identical, with a $5 \mathrm{~m}^{2}$ mirror collecting light onto an array of 256 phototubes. Each phototube covers about one square degree of the sky.

\section{Monocular Analysis}

The two detectors are designed to observe cosmic ray showers steroscopically. However, HiResI has been running for much longer than HiRes-II and at energies below about $10^{18} \mathrm{eV}$, events can only be observed by one detector. Thus we are presenting here spectra from monocular analyses.

Determination of the shower geometry in monocular mode is done by fitting the trigger times, $t_{i}$, of hits to the following function of the angles, $\chi_{i}$, of hits above the horizon,within the event plane:

$t_{i}=t_{0}+\frac{R_{p}}{c} \tan \left(\frac{\pi-\psi-\chi_{i}}{2}\right)$

Here $R_{p}$ is the impact parameter, $\psi$ the in-plane angle between the shower and the horizon, and $t_{0}$ the time of closest approach.

With limited elevation coverage, HiRes-I monocular events are too short in angular spread for reliable determination of $\psi$ and $R_{p}$ by timing alone. For this analysis, the expected form of shower development itself was used to constrain the time fit to yield realistic geometries. The shower profile was assumed to be described by the Gaisser-Hillas parameterization

$N(x)=N_{m}\left(\frac{x-x_{0}}{x_{m}-x_{0}}\right)^{\left(x_{m}-x_{0}\right) / \lambda} e^{\left(x_{m}-x\right) / \lambda}$

where $N(x), N_{m}$ are the number of particles at depth $x$ and at shower maximum depth $x_{m}$, respectively. The first-interaction depth and shower elongation constant are denoted by $x_{0}$, and $\lambda$ [3].This technique is called the profileconstrained fit (PCF). Equation 2 is in agreement with previous HiRes measurements [4, and with CORSIKA/QGSJET simulations 15 7]. Based on these, we fixed $x_{0}$ and $\lambda$ at 40 and $70 \mathrm{~g} / \mathrm{cm}^{2}$, respectively. We allowed $x_{m}$ to vary in $35 \mathrm{~g} / \mathrm{cm}^{2}$ steps between 680 and $900 \mathrm{~g} / \mathrm{cm}^{2}$, matching the 
expected range for proton to iron primaries in this energy range. This procedure breaks down for events with energies less than $3 \times 10^{18} \mathrm{eV}$, providing the lower limit to the HiRes-I spectrum.

The greater elevation coverage of HiRes-II allows for the reconstruction of the shower geometry from timing alone. Equation 1 is linear in $R_{p}$ and $t_{0}$, we find the best value for these variables analytically for each $\psi$; the best $\psi$ is then found by $\chi^{2}$ minimization. With the geometry of the shower known, we fit the observed light signal to the Gaisser-Hillas parameterization of Eqn. 2. We collected photo-electrons from all tubes into a sequence of time bins. This exploited the FADC data acquisition system and lessened our sensitivity to PMT acceptance.

Two calibration issues effect both sites: the absolute calibration of the phototube gains and the determination of the transparency of the atmosphere. The phototube gains are determined through a nightly illumination of the phototube clusters with a YAG laser and a monthly illumination by a Xenon flash bulb. The atmospheric transparency is determined by probing the atmosphere hourly with a set of laser shots from each of the sites which is observed and reconstructed by the other site.

\section{Data-MC Comparisons}

The aperture as a function of energy is a necessary component of the spectrum calculation. It is determined by Monte Carlo calculation. To verify our calculated aperture and analysis procedures, we undertook an extensive program of Monte Carlo simulation development and comparison of simulated data with real data. Two figures exemplify this; both are from the HiResII analysis. In figure 11 we compare data and MC distributions of two geometric variables: $r_{p}$ and $\psi$. In figure 2 we compare the distributions of reconstructed energies. Note that any large discrepancy between the atmospheric parameters used in $\mathrm{MC}$ and the conditions under which the data were actually taken would heavily influence the distribution of impact parameters.
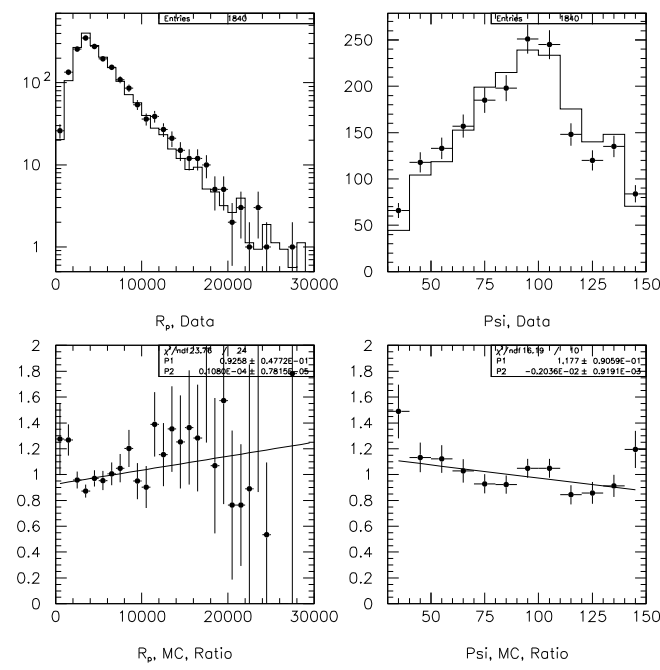

Figure 1. Data/MC comparisons of $r_{p}$ and $\psi$.

\section{The HiRes Spectra}

The two HiRes monocular spectra are shown in figure 3 [8,9] along with what one would expect from a model combining galactic sources with uniformly distributed extra-galactic sources 10]. The GZK cutoff is evident, and there is no need from this data to invoke extraordinary means in order to evade it.

In figure 4 we compare our spectra with that of AGASA [11]. The shape of the two spectra agree quite well for energies below $100 \mathrm{EeV}$, and reducing the AGASA energies by $20 \%$, which is within AGASA's stated systematic uncertainty, makes this comparison explicit. The famed discrepancy between HiRes and AGASA has thus been reduced to a few points above $100 \mathrm{EeV}$, where AGASA has five events (with they're energy scale reduced by the 20\%) and HiRes has only one.

\section{REFERENCES}

1. T. Abu-Zayyad et al., Proc. 26th Int. Cosmic Ray Conf. (Salt Lake City), 5, 349 (1999). 

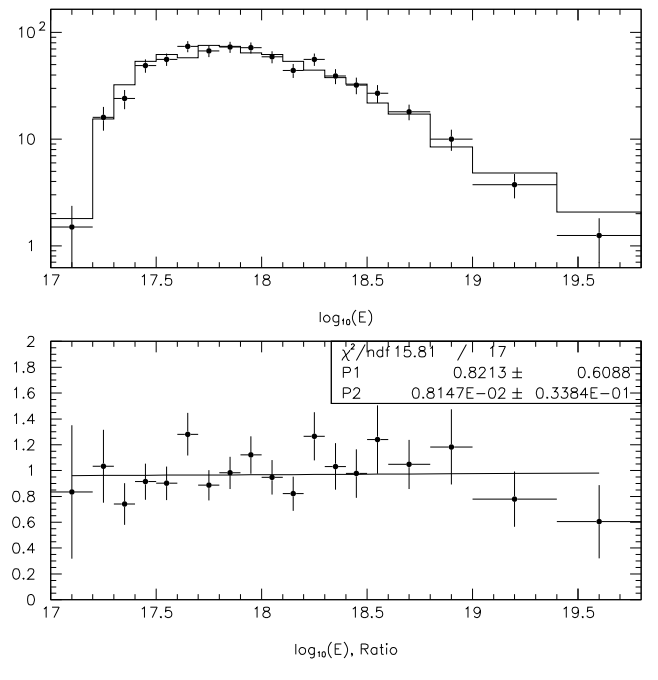

Figure 2. Data/MC comparison of reconstructed energies.

2. J. Boyer, B. Knapp, E. Mannel, and M. Seman, Nucl. Instr. Meth. A482, 457 (2002).

3. T. Gaisser and A.M. Hillas, Proc. 15th Int. Cosmic Ray Conf. (Plovdiv), 8, 353, (1977).

4. T. Abu-Zayyad et al., Astropart. Phys. 16, 1, (2001).

5. C. Song, Z. Cao et al., Astropart. Phys. 14, $7,(2000)$.

6. D. Heck, J. Knapp, J.N. Capdevielle, G. Schatz and T. Thouw "CORSIKA : A Monte Carlo Code to Simulate Extensive Air Showers", Report FZKA 6019 (1998), Forschungszentrum Karlsruhe.

7. N.N. Kalmykov, S.S. Ostapchenko and A.I. Pavlov, Nucl. Phys. B (Proc. Suppl.) 52B, 17, (1997).

8. T. Abu-Zayyad et al., submitted to Phys. Rev. Lett., astro-ph/0208243.

9. T. Abu-Zayyad et al., submitted to Astropart. Phys., astro-ph/0208301.

10. V. Berezinsky, A.Z. Gazizov, S.I Grigorieva, hep-ph/0204357.

11. M. Teshima, private communication.

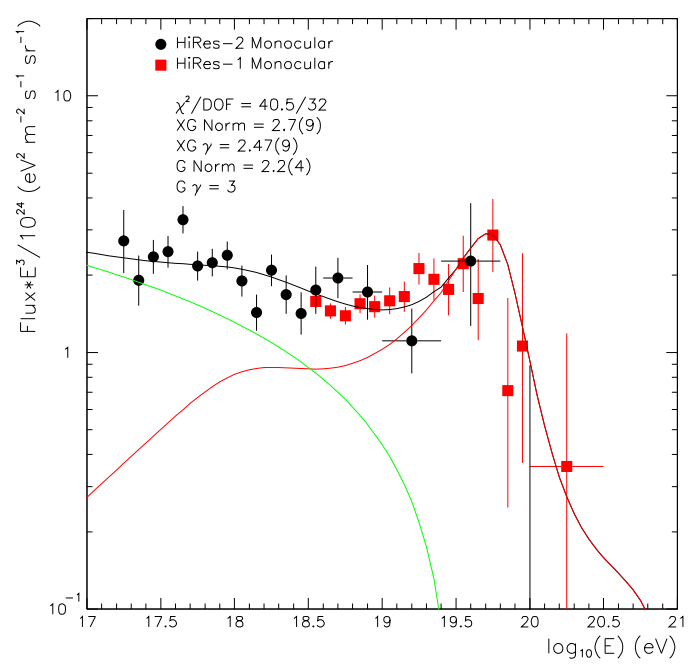

Figure 3. HiRes-I and HiRes-II spectra fit to galactic and uniform extragalactic source model.

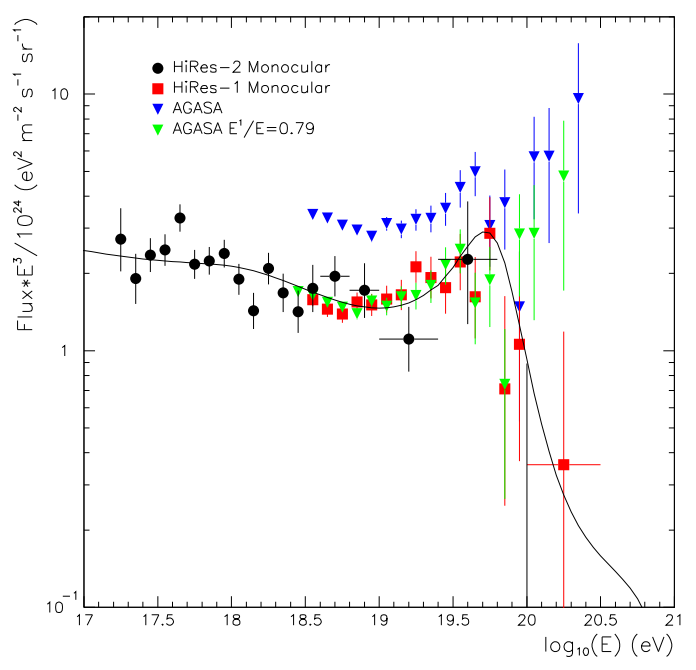

Figure 4. HiRes-I and HiRes-II spectra compared with AGASA. 